\title{
A covering theorem for families of sets in $\mathbb{R}^{d}$
}

\author{
John E. Wetzel and Wacharin Wichiramala*
}

\begin{abstract}
Let $\mathfrak{D}$ and $\mathfrak{F}$ be families of bounded sets in $\mathbb{R}^{d}, d \geq 2$. We describe a sense in which $\mathfrak{D}$ approximately covers $\mathfrak{F}$, and we show that then a compact set $X$ that covers $\mathfrak{D}$ must also cover $\mathfrak{F}$. As an application we show in the context of Moser's "worm" problem that a compact set in $\mathbb{R}^{d}$ that contains a congruent copy of every simple polygonal unit arc is a cover for the family of all unit arcs.
\end{abstract}

More than 40 years ago Leo Moser [7] (reprinted in W. Moser [8]) asked for the set of least area in the plane that can cover every planar arc of unit length. A great variety of similar problems can be formulated by restricting the covering set to be convex, or of prescribed shape (e.g., triangular, etc.), by seeking to minimize the perimeter, or thickness, or some other geometric measure, or by restricting the family of unit arcs in various ways (to be closed, or polygonal, or polygonal with at most $n$ segments, etc.). Very few of these problems, known collectively as "worm" problems, have been solved, and the analogous problems in higher dimensions are virtually unexplored. For a recent survey of the status of Moser's original problem in the plane, see Wetzel [11], and for a glimpse at the closely related notion of "escape" path, see Finch and Wetzel [4].

In this article we establish a covering theorem for families of bounded sets in $\mathbb{R}^{d}$, and we apply it to prove a covering approximation lemma that is potentially useful in "worm" problems in higher dimensions.

\section{The covering theorem in $\mathbb{R}^{d}$}

We suppose throughout that $d \geq 2$, and we begin by recalling the definition of the Hausdorff metric. Write $B_{\varepsilon}(x)$ for the open ball of radius $\varepsilon$ and center $x, U_{\varepsilon}(S)$ for the $\varepsilon$-neighborhood of the non-empty set $S$ (the set of points within $\varepsilon$ of some point of $S)$, and $\mathfrak{h}(A, B)$ for the Hausdorff distance between the non-empty sets $A$ and $B$, namely,

$$
\mathfrak{h}(A, B)=\operatorname{glb}\left\{r>0: \quad A \subseteq U_{r}(B) \text { and } B \subseteq U_{r}(A)\right\} .
$$

\footnotetext{
*Supported in part by the Thailand Research Fund (grant number MRG50800128).
} 
The Hausdorff distance is a metric on the family of all closed sets in $\mathbb{R}^{d}$ (see, for example, Burago et al. [2, p. 252] or Kelly and Weiss [5, Th. 8, p. 251-52]).

Two compact sets in $\mathbb{R}^{d}$ are congruent if there is an isometry of $\mathbb{R}^{d}$ that carries one onto the other. Convergence in the Hausdorff metric preserves congruence. Detailed proofs can be found in [5, Th. 8, p. 251-52] and Radunović [10], but for the sake of completeness, we supply a short proof of this fact pointed out to us by John Mackay. As usual, let $\mathbb{N}$ be the set of positive integers.

Lemma 1. Let $\left\langle S_{n}\right\rangle_{n=1}^{\infty}$ be a sequence of compact sets in $\mathbb{R}^{d}$ each of which is isometric to a set $S_{0}$. If $\lim _{n \rightarrow \infty} \mathfrak{h}\left(S_{n}, S\right)=0$, then $S$ is also congruent to $S_{0}$.

Proof. For each $n \in \mathbb{N}$ let $\mu_{n}: S_{0} \longrightarrow S_{n}$ be an isometry. Write $\left.\mu\right|_{X}$ for the restriction of $\mu$ to the set $X$, so that for each $n,\left.\mu_{n}\right|_{S_{0}}$ is a distance-preserving mapping of the compact set $S_{0}$ onto $S_{n}$. The family $\left\{\left.\mu_{n}\right|_{S_{0}}: n \in \mathbb{N}\right\}$ is clearly uniformly bounded and equicontinuous, and it follows from the Arzela-Ascoli Theorem that it has a uniformly convergent subsequence whose limit $\varphi$ is a distance-preserving mapping of $S_{0}$ onto $S$. Now the existence of an isometry $\mu$ on $\mathbb{R}^{d}$ so that $\left.\mu\right|_{S_{0}}=\varphi$ follows from an extension theorem of Benz [1, p. 20].

The Blaschke Selection Theorem plays an important role in what follows.

Theorem (Blaschke Selection Theorem). A bounded sequence of compact sets in $\mathbb{R}^{d}$ has a subsequence that converges in the Hausdorff metric to a compact set.

For a proof, see, for example, [2, pp. 252-54] or [5, p. 237-39].

We say that a set $F$ fits in a set $X$ (or $X$ covers $F$ ) if there is an isometry $\mu$ so that $\left.\mu\right|_{F}(F) \subset X$. A set $X$ is a cover for a family $\mathfrak{F}$ of sets if it covers each set $F$ in $\mathfrak{F}$. If $\mathfrak{D}$ and $\mathfrak{F}$ are families of bounded sets, it would be natural to extend the definition to say that $\mathfrak{D}$ covers $\mathfrak{F}$ if for each $F$ in $\mathfrak{F}$ there is a $D$ in $\mathfrak{D}$ that covers $F$. It would then follow immediately that a set $X$ is a cover for a family $\mathfrak{F}$ if it covers a family $\mathfrak{D}$ that is itself a cover for $\mathfrak{F}$. But the same conclusion follows from a slightly weaker condition.

Definition 1. Let $\mathfrak{D}$ and $\mathfrak{F}$ be families of bounded sets in $R^{d}$. We say that $\mathfrak{D}$ is a near cover of $\mathfrak{F}$ (or $\mathfrak{D}$ nearly covers $\mathfrak{F}$ ) if for each set $F$ in $\mathfrak{F}$ and each $\varepsilon>0$ there is a set $D$ in $\mathfrak{D}$ whose $\varepsilon$-neighborhood $U_{\varepsilon}(D)$ covers $F$. 
Theorem 2 (The covering theorem). Let $X$ be a compact set and let $\mathfrak{F}$ and $\mathfrak{D}$ be families of bounded sets in $\mathbb{R}^{d}$. If $X$ is a cover for $\mathfrak{D}$ and if $\mathfrak{D}$ nearly covers $\mathfrak{F}$, then $X$ is a cover for $\mathfrak{F}$.

Proof. Suppose $X$ is a cover for $\mathfrak{D}$ and $\mathfrak{D}$ nearly covers $\mathfrak{F}$, and let $F$ be a set in $\mathfrak{F}$. We have to show that $F$ fits in $X$. For each $n \in \mathbb{N}$ there is a $D_{n} \in \mathfrak{D}$ so that $U_{1 / n}\left(D_{n}\right)$ covers $F$. Since $X$ is a cover for $\mathfrak{D}, D_{n}$ fits in $X$, and consequently $U_{1 / n}\left(D_{n}\right)$ fits in $U_{1 / n}(X)$ and so does $F$. Let $F_{n}$ be a congruent copy of $F$ that lies in $U_{1 / n}(X)$. Then $\overline{F_{n}} \subset U_{2 / n}(X) \subset U_{1}(X)$. According to the Blaschke Selection Theorem, the sequence $\left\langle\overline{F_{n}}\right\rangle_{n=1}^{\infty}$ has a subsequence that converges in the sense of the Hausdorff metric to a compact set $F_{0}$ that is congruent to $\bar{F}$ (by Lemma 1); and since $\left\langle U_{2 / n}(X)\right\rangle_{n=1}^{\infty}$ converges to $X$ it follows that $F_{0} \subset X$. Consequently $F$ fits in $X$.

\section{The simple approximation theorem}

An important lemma in curve covering problems in the plane asserts that a compact, convex set contains a congruent copy of each unit arc if and only if it contains a congruent copy of each simple polygonal unit arc. This result, which we inexplicably neglected to state in our article [6] (with Maki), is an easy corollary of an approximation theorem having a late $19^{\text {th }}$ century classical character that we established there for precisely this purpose. Here we drop the assumption of convexity and extend this covering result to compact sets in $\mathbb{R}^{d}$.

Write $l(\gamma)$ for the length of a (rectifiable) arc $\gamma$ in $\mathbb{R}^{d}$ and $p q$ for the Euclidean distance between points $p$ and $q$ in $\mathbb{R}^{d}$. We choose not to make a notational distinction between a curve $\gamma$ and its trace (the pointset that is its range), because the intended meaning is always clear from the context. We regard the terms curve, path, and arc as synonymous.

The result desired would follow from Theorem 2 if we establish that the family (of the traces) of all simple, polygonal unit arcs is nearly dense in the family (of the traces) of all unit arcs. This is the assertion of the following lemma.

Lemma 3 (The simple approximation theorem). Suppose $p$ and $q$ are different points in $\mathbb{R}^{d}$ and let $\gamma$ be an arc of length $L>0$ from $p$ to $q$. For each $\varepsilon>0$ there is a simple polygonal path $\pi$ from $p$ to $q$ such that (a) $L-\varepsilon<l(\pi) \leq L$, and (b) the trace of $\gamma$ lies in $U_{\varepsilon}(\pi)$.

Proof. The claim is trivial if $p q=L$, so we suppose that $p q<L$. The arguments for $d \geq 3$ mirror those presented for the plane case in [6, Theorem 
14]. Parameterize $\gamma$ by arclength, so that $\gamma:[0, L] \longrightarrow \mathbb{R}^{d}$ with $\gamma(0)=p$ and $\gamma(L)=q$, and let $\varepsilon>0$ be given. We claim first that there is a polygonal arc $\varpi$ from $p$ to $q$ such that

$$
\left.\begin{array}{r}
L-\frac{1}{2} \varepsilon<l(\varpi)<L, \\
\mathfrak{h}(\varpi, \gamma)<\frac{1}{2} \varepsilon .
\end{array}\right\}
$$

There is a partition $\left\langle 0=t_{0}, t_{1}, t_{2}, \ldots, t_{n}=L\right\rangle$ of $[0, L]$ so that the polygonal path

$$
\varpi=\left\langle v_{0} v_{1} v_{2} \ldots v_{n}\right\rangle,
$$

where $v_{i}=\gamma\left(t_{i}\right)$, joins $p$ to $q$, the length $l\left(\gamma_{i}\right)$ of each subarc $\gamma_{i}$ from $v_{i-1}$ to $v_{i}$ is less than $\frac{1}{2} \varepsilon$, and $L-\frac{1}{2} \varepsilon<l(\varpi) \leq L$. Evidently $l\left(\gamma_{i}\right) \geq v_{i-1} v_{i}$ for each $i=1,2, \ldots, n$. If $l(\varpi)=L$, then

$$
L=l(\gamma)=\sum_{i=1}^{n} l\left(\gamma_{i}\right) \geq \sum_{i=1}^{n} v_{i-1} v_{i}=l(\varpi)=L,
$$

and it follows that $\gamma_{i}=\overline{v_{i-1} v_{i}}$ for each $i$, and consequently $\gamma=\varpi$. Since $p q<L$, this polygonal arc has at least one vertex $v_{j}$ so that $v_{j-1}, v_{j}$, and $v_{j+1}$ are not collinear; and moving $v_{j}$ a bit toward $v_{j+1}$ keeping both $\gamma_{j-1}$ and $\gamma_{j}$ shorter than $\frac{1}{2} \varepsilon$ shortens $\varpi$. Hence with no loss of generality we may suppose $l(\varpi)<L$. Now, $\gamma_{i} \subset B_{\varepsilon / 2}\left(v_{i}\right)$, and consequently

$$
\gamma \subset \bigcup_{i=0}^{n} B_{\varepsilon / 2}\left(v_{i}\right) \subset U_{\varepsilon / 2}(\varpi) .
$$

To complete the argument we observe that there is a simple polygonal $\operatorname{arc} \pi$ from $p$ to $q$ that satisfies the conditions

$$
\left.\begin{array}{r}
|l(\pi)-l(\varpi)|<\min \left\{\frac{1}{2} \varepsilon, L-l(\varpi)\right\} \\
\mathfrak{h}(\pi, \varpi)<\frac{1}{2} \varepsilon,
\end{array}\right\}
$$

from which the conditions (a) and (b) follow. For $d=2$ the adjustments needed to obtain the nearby simple polygonal arc $\pi$ from $\varpi$ are described in $[6$, Theorem 14, p. 652]. The arguments are similar but easier when $d \geq$ 3. As an assemblage of line segments, the polygonal arc $\varpi$ can fail to be simple in only a few ways: a vertex might meet $\varpi$ someplace else, or two edges of $\varpi$ might have a point other than a vertex in common. In each 
case, moving a vertex with its incident edges can eliminate the unwanted intersection, and these adjustments can be made in such a way that the resulting simple polygonal arc $\pi$ joins $p$ to $q$, it remains close to $\varpi$, and its length remains less than $L$. Now the claims of the theorem follow from the triangle inequality.

Here is the main consequence.

Theorem 4. The family $\mathfrak{D}$ of simple polygonal arcs of length at most $L$ nearly covers the family $\mathfrak{F}$ of all arcs of length $L$.

Proof. Let $\gamma$ be an arc of length $L$ from $p$ to $q$, and suppose $\varepsilon>0$ is given. If $p \neq q$, Lemma 3 provides the desired $\operatorname{arc} \pi$ in $\mathfrak{D}$ so that $\gamma$ lies in an $\varepsilon$-neighborhood of $\pi$.

If $p=q$, suppose that $\gamma$ is parameterized on arclength, and let $t^{\prime}$ be a real number so that $\gamma\left(t^{\prime}\right) \neq q$ and $L-\varepsilon<t^{\prime}<L$. Let $q^{\prime}=\gamma\left(t^{\prime}\right)$, and let $\gamma^{\prime}$ be the subarc of $\gamma$ from $p$ to $q^{\prime}$ and $\gamma^{\prime \prime}$ the subarc of $\gamma$ from $q^{\prime}$ to $q$, so that $\gamma=\gamma^{\prime} \oplus \gamma^{\prime \prime}$. Note that $l\left(\gamma^{\prime}\right)=t^{\prime}$. According to Lemma 3 there is a simple, polygonal arc $\pi$ from $p$ to $q^{\prime}$ such that $t^{\prime}-\varepsilon<l(\pi) \leq t^{\prime}$, and $\gamma^{\prime} \subset U_{\varepsilon}(\pi)$. Then $l(\pi)<L$ and both $\gamma^{\prime} \subset U_{\varepsilon}(\pi)$ and $\gamma^{\prime \prime} \subset B_{\varepsilon}\left(q^{\prime}\right) \subset U_{\varepsilon}(\pi)$. Consequently $\gamma \subset U_{\varepsilon}(\pi)$, as needed.

Corollary 5. A compact set in $\mathbb{R}^{d}$ contains a congruent copy of each arc of length $L$ in $\mathbb{R}^{d}$ if and only if it contains a congruent copy of each simple polygonal arc of length $L$ in $\mathbb{R}^{d}$.

Proof. Immediate from the Theorem, the preceding Lemma, and the observation that every polygonal arc of length at most $L$ is a subarc of a polygonal arc of length $L$.

Note that this Corollary fills a small hiatus in [9], where a certain compact but not convex set in the plane is claimed to be a cover for the family of all planar unit arcs because it contains a congruent copy of every simple planar polygonal unit arc.

\section{Acknowledgements}

It is a pleasure to acknowledge some useful conversations on this material with John Mackay at the University of Illinois. 


\section{References}

[1] Walter Benz, "Extensions of distance preserving mappings in euclidean and hyperbolic geometry," J. Geom. 79 (2004) 19-26. MR2041679

[2] Dmitri Burago, Yuri Burago, and Sergei Ivanov, A Course in Metric Geometry, Graduate Studies in Math. Vol. 33, American Mathematical Society, 2001. MR1835418

[3] Hallard Croft, Kenneth Falconer, and Richard Guy, Unsolved Problems in Geometry, Springer-Verlag, New York, 1991. MR1107516

[4] Steven R. Finch and John E. Wetzel, "Lost in a forest," Amer. Math. Monthly 111 (2004) 645-654. MR2091541

[5] Paul J. Kelly and Max L. Weiss, Geometry and Convexity: A Study in Mathematical Methods, John Wiley \& Sons, New York, 1979. MR0534615

[6] John M. Maki, John E. Wetzel, and Wacharin Wichiramala, "Drapeability," Discrete Comput. Geom. 34 (2005) 637-657. MR2173931

[7] Leo Moser, "Poorly formulated unsolved problems in combinatorial geometry," mimeographed (undated, but about 1966).

[8] W. O. J. Moser, "Problems, problems, problems," Discrete Appl. Math. 31 (1991) 201-225. MR1106701

[9] Rick Norwood and George Poole, "An improved upper bound for Leo Moser's worm problem," Discrete Comput. Geom. 29 (2003) 409-417. MR1961007

[10] Božidar Radunović, "Convergence in Hausdorff metric preserves geometric shape," Mat. Vesnik 48 (1996) 25-28. MR1410669

[11] "The classical worm problem - a status report," Geombinatorics XV (2005) 34-42; "Letter to the Editor," 92-93. MR2292763

John E. WeTZEL

UNIVERSiTy OF ILLINOIS AT URBANA-ChAMPAIGN

1409 West Green Street

URBANA, IL 61801

E-mail address: jewetz@comcast.net 


\author{
WACHARIN WICHIRAMALA \\ Department of Mathematics \\ FaCUlty of SCIENCE \\ Chulalongkorn University \\ BANGKOK 10330, THAILAND \\ E-mail address: wacharin.w@chula.ac.th
}

Received August 19, 2009 